\title{
Fuzzy Measures of Symmetry Breaking of Conditions, Similarity and Comparison: Non Statistical Information for the Single Patient
}

\author{
Cathy M. Helgason ${ }^{*}$ and Thomas H. Jobe
}

Department of Neurology, University of Illinois, College of Medicine, Chicago, Il 60612, USA

\begin{abstract}
Background: Science relies on experimentation to find truth. It demands that conditions remain unchanged for each repetition of an experiment. Thus, medicine relies on 'probability theory' based statistics and large double blind controlled randomized clinical trials. The purpose of this study is to discover measures that account for different and changing conditions of individual patients. These measures allow experiments to be performed without uniform conditions. They also allow precise prediction for the individual case. Methods: Variables of different patients or the same patient at different times are measured and normalized or expertly assigned a value in the unit interval to form the elements of a fuzzy "set as point" in the unit hypercube. Measures of breaking of symmetry of conditions, similarity, and comparison for different patient states are defined by fuzzy Subsethood measured in fuzzy cardinality. Fuzzy entropy measures for similarity and symmetry are discovered through the fuzzy Entropy theorem. Results and Conclusion: Measures of precise prediction for the single case and comparison of individual patient states capture the non linear dynamic between changing measured variables and symmetry of conditions. Non statistical information measures for this dynamic are discovered using the unifying structure of fuzzy theory and its measure space.
\end{abstract}

\section{INTRODUCTION}

One of the most important concepts in science is experimentation. Its unifying structure is what we here formally designate 'symmetry of conditions'. In this paper, a fuzzy measure of breaking of symmetry of conditions is related to those of similarity, comparison, and prediction [1]. This discussion proceeds in the setting of experimental and clinical medicine, but may have broader implications for the biological sciences in general. For precise and consistent analysis, all of these measures are defined by fuzzy Subsethood measured in fuzzy cardinality $[2,3]$. Fuzzy Subsethood and cardinality have been called the "fundamental unifying structure" and "measure space" of fuzzy theory $[2,4]$. In this study, they provide the framework within which study of the single patient at different states over time and comparison between distinct patients can occur.

Symmetry of conditions refers to the concept that at repeated trials in any experiment, the conditions of the experiment remain unchanged. Conditions in this regard refer to all variables or elements present in the universe, other than the ones being measured, a change in which might affect experimental results. These other elements must be kept constant in order to insure their neutrality in the calculation of causation in the measured variables. This type of symmetry of conditions is satisfied by probability theory and its statistical translation to experimental medicine [5]. Geometrically, a definition of symmetry of conditions is found in the unit hypercube where on each unit interval there is a symmetry about the point $\{0.5\}$, and in the unit hypercube of any number of axes, maximal about the midpoint $[6,7]$. This is symmetry of element values. Another kind of symmetry is related to fuzzy cardinality, the size of fuzzy sets as points.

\footnotetext{
*Address correspondence to this author at the Department of Neurology, University of Illinois, College of Medicine, Chicago, Il 60612, USA;

E-mail: helgasonkhooby@gmail.com
}

Breaking of symmetry of conditions implies a distortion of the geometry of conditions.

Using fuzzy Subsethood and fuzzy cardinality a measure of breaking of symmetry of conditions has previously been defined by us and named ' $\mathrm{K}$ ' [1]. A measure for breaking of symmetry of conditions allows causality to account for not only interaction between measured specified elements, but between these and their surrounding conditions.

Practically, comparison between patients drives clinical diagnosis and treatment for any one given patient, as in the expert decision of a physician. On the other hand, comparison to patients in a large double blind controlled randomized clinical study may apply when expertise is absent or in response to' guidelines'. Over a period of time, as when a physician follows and treats a patient for a chronic condition, both measured elements of the patient as well as his conditions are constantly changing. Therefore, complexity demands cognitive acumen in clinical judgment. This cognitive ability is built on perception.

The complexity of the clinical situation and expertise of the clinician are considered ambiguous and inscrutable because they have hitherto not been precisely represented by numbers. As we demonstrate in this paper, the dynamic of a patient's changing clinical state within changing conditions can be precisely represented using some tools of the Generalized Theory of Uncertainty, more specifically those of fuzzy logic and more generally fuzzy theory [6-8].

\section{METHODS AND MATERIALS}

We hypothesized that (a) prediction for the single case, as in a single patient, and (b) comparison of different states of a single patient over time or of different patients are both possible using the fundamental unifying concept and measure space of fuzzy theory. These are fuzzy subsethood and fuzzy cardinality. Specifically, fuzzy measures of similarity, 
comparison, and prediction can be expressed in the same units and account for not only measured specified variables of a patient, but also for different or changing conditions. The dynamic of change in one patient's state over time can be captured by fuzzy entropy.

In this study we employ the following tools for these measurements: graduation, granulation, fuzzy sets, fuzzy 'sets as points', fuzzy cardinality, fuzzy Subsethood, and fuzzy entropy [2-4, 6-11]. The key to defining the measures of fuzzy similarity, comparison, and entropy of symmetry of conditions is the measure for breaking of symmetry of conditions ' $\mathrm{K}$ ' defined in our previous work [1, 12].

For those unfamiliar with fuzzy sets, fuzzy cardinality, the fuzzy Subsethood theorem, the fuzzy unit hypercube, 'sets as points', and fuzzy entropy, these are now defined with other terms as they are used in this paper.

Fuzzy theory allows everything to be to a degree. Fuzzy sets, first defined by Zadeh, define set membership to a degree $[6,8]$. Each element of the set is allowed to be present to a degree and to take on a value in the unit interval without any rules confining the value that each set element can take on in relation to the others. When representing a patient as a fuzzy set the value an element takes on is arrived at by one of two methods: 1) by measurement, say in the laboratory, followed by normalization, or 2) by expert assignment of a value to the unit interval. Examples of elements in a patient are physiologic parameters, clinical states, laboratory test results, and so forth. When such elements represent a patient in a fuzzy set, they represent that patient's state as expressed through those elements.

Graduation, allowing an element to be present to a degree, is a cognitive tool of the physician [7]. Granulation, also a cognitive tool, allows the expertise of a physician to assign clumps of values to a variable such that these values are similar in nature [7]. An example of granulation is disability after stroke: "Very much", "moderate", "slight", "none" where a range of values applies to each granule and overlapping is allowed. Graduation and granulation allow expert perceptions to take on precise values. They are tools of fuzzy logic, the logic of the Generalized Theory of Uncertainty [6].

Fuzzy cardinality is the fundamental measure space of fuzzy theory [2]. The fuzzy cardinality of a fuzzy set A is symbolically represented by $M$ (A), where ' $M$ ' is read as 'the measure of'. It is equal to the sum of the values of the elements of A. Geometrically, it is the magnitude of a vector drawn from the origin of a unit hypercube to the fuzzy set A $[2,7]$. Being the sum of the elements of the fuzzy set of interest, it can be viewed as the 'size' of the fuzzy set. Size is an important concept for demonstration of symmetry and breaking of symmetry of conditions, because it means that no two fuzzy sets are exchangeable in 'size' unless their elements sum to the same value.

The fuzzy Subsethood theorem was derived both algebraically and geometrically by Kosko who designated it the fundamental unifying structure in fuzzy theory [4]. Given two fuzzy sets $\mathrm{A}$ and $\mathrm{B}$, it defines the degree to which one set belongs to another. It is expressed by the formula $\mathrm{S}$ (A, $\mathrm{B})=\mathrm{M}(\mathrm{A} \cap \mathrm{B}) / \mathrm{M}(\mathrm{A})$ which is read "the degree to which $\mathrm{A}$ is a subset of $B$ '. The symbol ' $\cap$ ' is read as 'overlap' or 'in- tersect', and when two fuzzy sets are compared, it commands one to take the lesser of the elements being compared. So the fuzzy intersection of $\{0.2,0.3\}$ and $\{0.4,0.1\}$ is $\{0.2,0.1\}$.

Lotfi Zadeh first suggested that fuzzy sets can be represented geometrically in a unit hypercube of any number of axes, each valued in the unit interval $[1,7]$. Each axis represents a fuzzy set element. This representation has the advantage of visualization and demonstration [10, 13]. Visualization allows the transformation of fuzzy 'set as point' A to B to become representative of the changing state of a patient over time or for two fuzzy sets as points A and B to represent different patients and their difference to be perceived within different symmetries of conditions [10]. We have previously described how stroke patients can be so represented [9]. The unit hypercube also allows visualization of the probability space where the sum of the elements of each fuzzy set must add up to 1 [11]. Thus, in the probability space, each fuzzy set is of the same 'size' or fuzzy cardinality. Sets in the probability space can be exchanged with no change in symmetry of conditions of that space. Symmetry of conditions in this instance is symmetry of cardinality. This can be demonstrated by envisioning each fuzzy set as a ball of certain size. In the probability space the fuzzy cardinality of all sets is uniform. Elsewhere in the unit hypercube where fuzzy sets are of different 'size' or fuzzy cardinality, this is not the case and the exchange of any two fuzzy sets of different size or cardinality disturbs the relative position of all other sets.

The measure of breaking of symmetry of conditions ' $\mathrm{K}$ ', previously designated by us a causation measure, is defined from the fuzzy Subsethood theorem [1,4]. It represents the role of unknown unspecified variable elements in causing the transformation of one fuzzy set to another, as when fuzzy 'set as point' A transforms to fuzzy 'set as point' B. We demonstrated using continuous cellular automata that ' $\mathrm{K}$ ' is a measure of symmetry breaking [12]. On the other hand, its reciprocal $1 / \mathrm{K}$ restores symmetry to a certain degree. The measure ' $\mathrm{K}$ ' lies outside the unit interval, but $1 / \mathrm{K}$ within the unit interval.

Precisiation is a term which is used by Zadeh and generally means to give precision to otherwise ambiguous or vague percepts and concepts [6]. More specifically, it is a term used for computation with natural language, a unique capability of the Generalized Theory of Uncertainty [6]. In this paper, we employ this term in its more general sense. Dimension is the term used herein for all those elements, objects, attributes of interest specified or unspecified. Dimensions are either measured or unmeasured and represent a manifold for an attribute, object or element of interest. The type of measure used on the manifold is the metric for that dimension. Fuzzy logic uses the real line unit interval 0 to 1 as it's metric. Fuzzy similarity, comparison and prediction are represented in dimensional units. We consider the cardinality of a fuzzy set itself to be a measure of dimensionality of that set. Each axis of the unit hypercube is a dimension. When dimensions are unspecified and unmeasured they are referred to as "unknown" and collectively referred to as "the unknown". The exchange of two fuzzy sets of same or different cardinality is often referred to by us as transformation of fuzzy set A to fuzzy set B. The term transformation un- 
dercores the dynamic of the exchange. The comparison of two fuzzy sets behaves as an exchange.

The symbol " $n$ " refers to the number of elements in the fuzzy set or the number of axes of the unit hypercube. More generally, it is the number of 'dimensions' as defined previously.

The symbol $\mathrm{Z}$ refers to the number of fuzzy sets to be compared.

The symbol E refers to the fuzzy entropy of a fuzzy set. It measures information. It tells us how indiscernible two objects of complementary value are. The more indistinguishable, the more information. So, the more two patients are similar, the more the physician can expect they may behave diagnostically and therapeutically alike. A measure of discernability helps the physician in pattern matching to memories of previous patients or between two different patients or the same patient's different states over time. In this study, patients are compared in their known measured elements as well as their different conditions. We define an entropy measure for these and call them respectively fuzzy entropy of similarity and fuzzy entropy of symmetry. The fuzzy entropy theorem is defined by Kosko and is expressed by $\mathrm{E}$ (A), where $A$ is a fuzzy set [14]. It is expressed as $E(A)=M$ $\left(A \cap A^{c}\right) / M\left(A U A^{c}\right)$, where $A^{c}$ is the complement of $A$. The symbol ' $U$ ' is read as union, and commands one to take the larger of the element values. The union of fuzzy sets $\{0.3,0.4\}$ and $\{0.7,0.2\}$ is $\{0.7,0.4\}$.

Complementarity ' $c$ ' is a basic fuzzy set operation. The complement of a fuzzy element $\{a\}$ is $\{1-a\}$ [8] Because fuzzy logic violates the Aristotelian and bivalent laws of identity, the excluded middle and non contradiction, the sum of $a$ and its complement do not make up the whole universe and the intersection of the element with its complement is not the null set. Probability theory relies on binary Aristotelian logic. The Generalized Theory of Uncertainty relies on fuzzy logic [6].

In this paper, symmetry refers to that which does not change in the midst of change [15]. Symmetry breaking refers to the fact that the things previously thought would not change actually do change. The measure ' $\mathrm{K}$ ' is the fuzzy measure of breaking of symmetry of conditions [1]. It is defined using fuzzy Subsethood and cardinality and is expressed by the following:

$$
\mathrm{K}=\frac{\sqrt{(\mathrm{M}(\mathrm{A} \cap \mathrm{B}) / \mathrm{M}(\mathrm{A}))} \times \sqrt{(\mathrm{M}(\mathrm{A} \cap \mathrm{B}) / \mathrm{M}(\mathrm{B})) /}}{(\mathrm{M}(\mathrm{A} \cap \mathrm{B}) / \mathrm{M}(\mathrm{A}))^{2} \mathrm{x}(\mathrm{M}(\mathrm{A} \cap \mathrm{B}) / \mathrm{M}(\mathrm{B}))^{2}}
$$

The measure $\mathrm{K}$ breaks the unit interval and $1 / \mathrm{K}$ restores it. They do this to a certain extent. The measure $\mathrm{K}$ lies outside the unit interval. Breaking of symmetry of conditions allows a topological distortion of fuzzy sets as points in the unit hypercube. In the probability space this is such that the geometry of the sets remains unchanged and no topological distortion takes place. Otherwise, the distortion creates a new curvature of the fuzzy hypercube space. This again can be demonstrated by considering the exchange of two fuzzy sets as points of different size when these sets are balls of different size. An exchange of balls of the same size does not affect the position of any other ball in the unit hypercube, but an exchange of balls of different size affects all of them. An entropy measure for the tendency to break and restore conditions is defined using the fuzzy entropy theorem.

\section{RESULTS}

\section{A Fuzzy Measure of Similarity}

In order to use the same units of measure and underlying geometry as K, the fuzzy measure for breaking of symmetry of conditions, we define a fuzzy measure of similarity from fuzzy Subsethood measured in fuzzy cardinality.

Fuzzy similarity (F Sim (A,B)) of fuzzy sets and visualized as sets as points in the unit fuzzy hypercube, A and B, can be represented by the following expression: FSim $(\mathrm{A}, \mathrm{B})=\mathrm{n} / \mathrm{Z}(\mathrm{S}(\mathrm{A}, \mathrm{B})+\mathrm{S}(\mathrm{B}, \mathrm{A}))$, where $\mathrm{S}(\mathrm{A}, \mathrm{B})$ and $\mathrm{S}(\mathrm{B}, \mathrm{A})$ are the fuzzy subsethood measures of $\mathrm{A}$ in $\mathrm{B}$ and $\mathrm{B}$ in $\mathrm{A}$, otherwise stated as all of those sets of A which belong to B to a degree and all of those of $\mathrm{B}$ which belong to $\mathrm{A}$ to a degree. Sets here refer to 'sets as points' in the unit hypercube. The variable ' $n$ ' refers to the number of dimensions of the unit hypercube at stake. This is the same as the number of elements in each fuzzy set. The variable ' $Z$ ' is the number of fuzzy sets to be compared. The variables $\mathrm{n}$ and $\mathrm{Z}$ allow the fuzzy subsethood of A in B and B in A to be distributed amongst the elements. It tells us how many elements share the subsethood between $\mathrm{A}$ and $\mathrm{B}$. Thus, FSim $(\mathrm{A}, \mathrm{B})=\mathrm{n} / \mathrm{Z}$ $((\mathrm{M}(\mathrm{A} \cap \mathrm{B}) / \mathrm{M}(\mathrm{A}))+(\mathrm{M}(\mathrm{A} \cap \mathrm{B}) / \mathrm{M}(\mathrm{B}))$, where $\mathrm{M}$ is fuzzy cardinality or the sum of the elements of fuzzy sets A or B and subsethood is defined by the fuzzy Subsethood theorem [4]. Similarity of course does not have to be measured at the level of the elements, in which case $n / Z$ does not have to be included in its expression. The ability to measure similarity at the level of elements allows two fuzzy sets of different number of elements to be compared.

The fuzzy measure of similarity lies outside the unit interval. That is in part because it subsumes within it the different symmetries of conditions of the fuzzy sets being compared.

\section{Fuzzy Measures of Comparison and Predictability}

Given the fuzzy measures of similarity (FSim A,B) and breaking of symmetry of conditions $(\mathrm{K})$, we now can compare any two sets as points in the unit fuzzy hypercube and take into account both their elements and their different conditional context or symmetry. The fuzzy measure of comparison for two such fuzzy sets is defined by the following expression where FComp (A, B) is the fuzzy measure of comparison of fuzzy sets as points $A$ and $B$ :

FComp $(\mathrm{A}, \mathrm{B})=\mathrm{FSim}(\mathrm{A}, \mathrm{B})-(\mathrm{K}-1))$, and can be read as the similarity of fuzzy sets A and B taking into account the different symmetry of conditions for A and B. It is a measure of how similar the sets are when their different conditions are removed. It is a measure of how different the number of elements sharing the subsethood between A and B becomes when difference in conditions are accounted for. One is subtracted from $\mathrm{K}$ because it is the value of $\mathrm{K}$ when breaking of symmetry of conditions does not take place, as in the probability space. This measure also lies outside the unit interval. This is in part because it still subsumes the restored or same symmetry of conditions. 
One can also calculate a fuzzy measure of predictability (FPred) for any action which caused the change from $\mathrm{A}$ to $\mathrm{B}$.

FPredict $(\mathrm{A}, \mathrm{B})=\mathrm{FComp}(\mathrm{A}, \mathrm{B}) /(\mathrm{FSim}(\mathrm{A}, \mathrm{B})$, to be read as to what degree is the similarity of $\mathrm{A}$ and $\mathrm{B}$, the number of elements sharing the subsethood of $\mathrm{A}$ in $\mathrm{B}$ and $\mathrm{B}$ in $\mathrm{A}$, changed taking into account breaking of symmetry of conditions, when A becomes B. What percentage of the similarity of $\mathrm{A}$ and $\mathrm{B}$ is due to their elements and like conditions alone. Stated otherwise, to what degree is the distribution of subsethood between A and B the same as it was before the change in conditions were accounted for or what percentage of similarity between $A$ and $B$ remains when the different conditions of $\mathrm{A}$ and $\mathrm{B}$ are excluded. It is this measure which defines for the physician the causal relationship between change in subsethood for the specified elements and a given intervention, while ignoring change in conditions and yet not confining the analysis to the rules of probability [9].

In the dimension of probability for a two dimensional fuzzy unit hypercube, the fuzzy similarity, the number of elements sharing subset hood of A and B and B in A changes depending on the $\mathrm{M}(\mathrm{A} \cap \mathrm{B})$ and $\mathrm{n} / \mathrm{Z}$. But, in probability, the fuzzy measure of breaking of symmetry of conditions $K$ is always 1 no matter what A and B are as fuzzy sets. The measure of fuzzy comparison, the number of elements sharing subsethood of $\mathrm{A}$ in $\mathrm{B}$ and $\mathrm{B}$ in $\mathrm{A}$ given the change in conditions is the same as the fuzzy measure of similarity in the probability space. This is because the effect of different symmetries of conditions is nil. The fuzzy measure of predictability in probability is 1 . It is the degree to which an intervention changed the number of elements sharing subsethood of $\mathrm{A}$ in $\mathrm{B}$ and $\mathrm{B}$ in $\mathrm{A}$ given the change in conditions. This measure lies in the unit interval, and so a predictability measure of 1 is highest or $100 \%$. That means the fuzzy similarity of $\mathrm{A}$ and $\mathrm{B}$ does not change depending on conditions and the intervention action alone accounted for all of the subsethood. No matter what A and B are as fuzzy sets, if they are in the probability space, predictability never changes. This is the power of using probability for measuring causation.

\section{Fuzzy entropy of similarity and Fuzzy Entropy of Sym- metry}

Fuzzy entropy refers to the uncertainty of a system or message [2]. In the case of interchange or transformation of two fuzzy sets, a change of entropy takes place which makes the fuzzy set of outcome either fuzzier or crisper in terms of the values of its elements. If more of those elements are close to 0.5 , then the set has become fuzzier. Information comes from the fuzziness or clarity of a set in this sense [2]. But the phyisician needs another kind of information, information concerning the distinguishability or indistinguishability of the fuzzy sets in question. How much does the patient now resemble what he was? How similar are these patients in their diagnosis and in their response to treatment? This information tells the physician the difference between fuzzy sets $\mathrm{A}$ and $\mathrm{B}$ with and without their different conditions.

When fuzzy set A goes to fuzzy set B there is a coincidental dynamic of fuzzy similiarity and breaking (with partial restoration) of symmetry of conditions. As more transformations between fuzzy sets occur over time, as $\mathrm{K}$ changes F (Sim) changes. Each K corresponds to the transformation of A to B as reflected by the fuzzy similarity measure. As the symmetry of conditions of A breaks and becomes that of $\mathrm{B}$, the degree to which A belongs to B and the degree to which $\mathrm{B}$ belongs to A changes. The entropy of this transformation dynamic can be measured. It tells you how much the fuzzy sets $\mathrm{A}$ and $\mathrm{B}$ are the same and how much they are different at the same time depending on a taking into account their different symmetries of conditions. It is how different or the same the fuzzy measure of similarity is with and without different conditions.

The expression for what we now name fuzzy entropy of similarity is the following expression where fuzzy set elements are now $\{\mathrm{F} \mathrm{Sim} \mathrm{A}, \mathrm{B}\}$ and $\{\mathrm{F} \mathrm{Comp}\}$ :

$$
\begin{aligned}
& \mathrm{E}(\mathrm{F} \operatorname{Sim}(\mathrm{A}, \mathrm{B}), \mathrm{F} \operatorname{Sim}(\mathrm{A}, \mathrm{B})-(\mathrm{K}-1)) \\
& =\frac{\{\mathrm{F} \operatorname{Sim}(\mathrm{A}, \mathrm{B}), \mathrm{F} \operatorname{Sim} \mathrm{A}, \mathrm{B}-(\mathrm{K}-1)\} \cap\{\mathrm{F} \operatorname{Sim}(\mathrm{A}, \mathrm{B}), \mathrm{F} \operatorname{Sim}(\mathrm{A}, \mathrm{B})-(\mathrm{K}-1)\} \mathrm{c})}{\{\mathrm{F} \operatorname{Sim}(\mathrm{A}, \mathrm{B}), \mathrm{F} \operatorname{Sim}(\mathrm{A}, \mathrm{B})-(\mathrm{K}-1)\} \mathrm{U}\{\mathrm{F} \operatorname{Sim}(\mathrm{A}, \mathrm{B}), \mathrm{F} \operatorname{Sim}(\mathrm{A}, \mathrm{B})-(\mathrm{K}-1)\} \mathrm{c})}
\end{aligned}
$$

and where each element is normalized by $\mathrm{F} \operatorname{Sim}(\mathrm{A}, \mathrm{B})+\mathrm{F}$ $\operatorname{Sim}(\mathrm{A}, \mathrm{B})-(\mathrm{K}-1)$ allowing our fuzzy entropy of similarity measure to fall in the unit interval.

In this circumstance, fuzzy entropy of similarity always equals 1 when $F \operatorname{Sim}(A, B)$ and $F \operatorname{Sim}(A, B)-(K-1)$ are the same as is the case in the probability space where there is no change in symmetry of conditions and $\mathrm{K}$ always equals 1 . Entropy of similarity is 0 or near zero when the difference between $F \operatorname{Sim}(A, B)$ and $F \operatorname{Sim}(A, B)-(K-1)$ is maximum, where symmetry of conditions has changed the most. This goes along with the idea that when $\mathrm{K}$ is largest, the difference between $\mathrm{A}$ and $\mathrm{B}$ is greatest.

When $n / Z$ is included in the $F \operatorname{Sim}(A, B)$ definition, we have information regarding the number of elements carrying the subsethood between A and B. This never changes in the probability space. Elsewhere in the unit hypercube, if $\mathrm{E}$ is low then the number of elements carrying the subsethood between $\mathrm{A}$ and $\mathrm{B}$ is very different depending on conditions. Because our entropy measure is fuzzy, the maximum uncertainty about the role of conditions in accounting for the similarity between $\mathrm{A}$ and $\mathrm{B}$ is when conditions do not change. The clearest message about the role of conditions is when $\mathrm{K}$ is highest and the comparison between fuzzy sets is not confined to the probability space. Low entropy of similiarity, big role for symmetry breaking in accounting for subsethood between A and B. High E Sim (A,B), low role for conditions in accounting for subsethood between A and B. Clinically, when comparing two patients or a single patient's state over time, the higher the fuzzy entropy of similarity measure, the more the measured variables account for his change in state. The lower this entropy measure, the more something outside of those measured known variables and same symmetry of conditions plays a role.

Finally, as we have defined $\mathrm{K}$ and $1 / \mathrm{K}$ we need a measure of how these two compare for any one given transformation of fuzzy sets. Using again the general formula for fuzzy entropy and normalizing $\mathrm{K}$ and $1 / \mathrm{K}$ by $\mathrm{K}+1 / \mathrm{K}$ we are able to define an entropy of symmetry of conditions. This measure falls within the unit interval and tells us how much the tendency to break and restore symmetry compares to a measured tendency to do the same to the contrary. It tells us how the degree to which symmetry of conditions breakage equals that of its restoration. We will express this measure by $\mathrm{E}((\mathrm{K}$ 
$(\mathrm{A}, \mathrm{B}), 1 / \mathrm{K}(\mathrm{A}, \mathrm{B}))$ and call it Entropy of Symmetry. In the probability space this measure equals 1 . This is consistent with the complete restoration of symmetry of conditions when one fuzzy set transforms to another in the probability space.

$\mathrm{E}(\mathrm{K}(\mathrm{A}, \mathrm{B}), 1 / \mathrm{K}(\mathrm{A}, \mathrm{B}))$

$=\mathrm{M}\left(\{\mathrm{K}, 1 / \mathrm{K}\} \cap\{\mathrm{K}, 1 / \mathrm{K}\}^{\mathrm{c}}\right) / \mathrm{M}\left(\{\mathrm{K}, 1 / \mathrm{K}\} \mathrm{U}\{\mathrm{K}, 1 / \mathrm{K}\}^{\mathrm{C}}\right)$

Where $\mathrm{K}$ and $1 / \mathrm{K}$ are normalized by $\mathrm{K}+1 / \mathrm{K}$, and where $\mathrm{K}$ and $1 / \mathrm{K}$ correspond to the transformation or comparison of fuzzy sets as points A and B in the unit hypercube.

\section{Medical Experiments Designed to Give Information in the Form of Information Granule Using Measures of Fuzzy Entropy of Similarity and Fuzzy Entropy of Sym- metry}

We have provided the basic definitions for measures that are useful both in the clinical setting and in the experimental setting. We now give examples that will illustrate their use.

1) Comparison of the Clinical Patient to the Average Patient of a Large Double Blind Randomized Controlled Clinical Trial

First, the physician and the patient want to apply the statistical information available from large double blind controlled randomized clinical trials to that patient's particular case. With the use of the fuzzy measure of prediction we are able to compare the patient facing the physician to the average patient of any group study. The way this is done is the following:

Identify the question to be answered. What is the risk reduction our patient will have if he/she takes a certain medication for a particular condition? What are the chances he/she will die, have a stroke, or have a myocardial infarction if he takes the medication or if he/she does not? The usual difficulty the physician has in providing the patient with a precise answer to these questions is that the patient is somehow in some fashion to a degree different from the average patient of the scientific group study by which he/she is to provide the answer. The physician can not provide anything other than a probability of how the patient will do. This probability is based on Baysian inference but leaves some chance that the patient will not behave how the physician predicts.

The measure provided by the fuzzy prediction measure is not based on probability and is an exact measure of how the patient compares to the average patient of the clinical group study. Now let us give an example using a hypothetical patient. The patient in question is 80 years old, had a stroke 7 months ago and has been taking aspirin now for 10 months. He wants to know how effective his aspirin is compared to if he had been placed on clopidogrel. The etiology of his stroke was not found and so he fell within the cryptogenic stroke diagnosis, but he had known peripheral arterial disease and therefore known atheroma. The elements we want to focus on are those that are different in measure from the average patient of the study we want to compare him to.

In the Caprie study, a large double blind randomized controlled clinical trial of clopidogrel $v s$ aspirin for recurrent stroke prevention, the results showed an $8.7 \%$ overall risk reduction in favor of clopidogrel compared to aspirin. The percent of persons taking clopidogrel who suffered an outcome event of ischemic stroke was $4.6 \%$ and $4.8 \%$ of those on aspirin. The patients randomized had a stroke within 6 months of randomization, took study drug for an average of 1.6 years. The elements here for comparison are time since stroke placed on aspirin and total time on aspirin. For our example patient this is 7 months and 10 months. For the average study patient these numbers are 6 months and 18 months. The elements of our example patient and study patient must be normalized to form elements of a fuzzy set as point. We can normalize by 24 months. Thus our clinical patient who is named $\mathrm{A}$ is $\{0.29,0.42\}$ and the average $\mathrm{Ca}$ prie study patient $\mathrm{B}$ is $\{0.25,0.75\}$. We now have two patients as fuzzy sets as points to compare.

$$
\begin{aligned}
& \mathrm{F} \operatorname{Sim}(\mathrm{A}, \mathrm{B})=\mathrm{S}(\mathrm{A}, \mathrm{B})+\mathrm{S}(\mathrm{B}, \mathrm{A}) \\
& =M(A \cap B) / M(A)+M(B \cap A) / M(B) \\
& =\mathrm{M}\{0.25,0.42\} / \mathrm{M}\{0.29,0.42\}+\mathrm{M}\{0.25,0.42\} / \mathrm{M}\{0.25 \text {, } \\
& 0.75\} \\
& =0.67 / 0.71+0.67 / 1.0 \\
& =0.94+0.67 \\
& =1.61 \\
& \mathrm{~K}(\mathrm{~A}, \mathrm{~B})=\sqrt{0.94 \times 0.67} /(0.94 \times 0.67)^{2} \\
& =0.79 / 0.40 \\
& =1.98 \\
& \mathrm{~F} \operatorname{Comp}(\mathrm{A}, \mathrm{B})=\mathrm{F} \operatorname{Sim}(\mathrm{A}, \mathrm{B})-(\mathrm{K}-1) \\
& =1.61-(1.98-1) \\
& =1.61-0.98 \\
& =0.63 \\
& \text { F Pred }(\mathrm{A}, \mathrm{B})=\mathrm{F} \operatorname{Comp}(\mathrm{A}, \mathrm{B}) / \mathrm{F} \operatorname{Sim}(\mathrm{A}, \mathrm{B}) \\
& =0.39
\end{aligned}
$$

So, now we are ready to tell our patient that because he has been on aspirin, he has had for the time he has been on aspirin a $0.39 \times 4.6 \%=1.79 \%$ chance of having a stroke had he been on clopidogrel for this time compared to the $1.87 \%$ chance he has had because he has been on aspirin. (This is assuming a period of 6 months since time of stroke for the average Caprie study patient randomization.)

The information just presented is in non statistical form. It is a measure of how similar the clinical patient is to the average patient of the Caprie study, taking into account his different conditions.

\section{2) An Example of How to Conduct a Controlled Experi- ment with Results Reported in Granules of Fuzzy Informa- tion}

The information measures, based on fuzzy entropy, are granules of information about subsethood (similarity) and symmetry of conditions. This information belongs to the channels connecting any two fuzzy sets as points in the unit hypercube. As such they provide non statistical information. We now show how these measures can be used to test a study drug. This is an example of the application of our fuzzy measures of similarity, comparison and symmetry to 
experimental medicine. The information gained is in the form of fuzzy entropy measures of similarity and symmetry.

We can consider a study involving two groups of patients, one group that takes a study medication, and another that is given placebo. For each patient in the study, there is a baseline measure of elements of interest, and an endpoint measure of the same elements of interest. The baseline state of any given patient can be called $\mathrm{A}$, and the endpoint state B. Each patient during the study can be represented as a fuzzy set as point A and B. For each A to B, for each study subject, we can calculate know how much difference is due to change in the symmetry of conditions or change in elements. The elements are those targeted of interest in relation to study drug effect, for example. This information is non statistical in nature.

In another experimental setting we might want to use our measures so that they are relevant for a population of study subjects, some of whom are on study drug, and the others controls. In this experimental setting of patients and controls, each patient or control changes his elements and his conditions from baseline measurement $\mathrm{A}$ to outcome measurement B. We give the example of $n$ patients and $n$ controls, for $2 x n$ total study patients and 4xn "granules" of information being produced from that trial for each matched patient- control. Each granule is an information measure, measured in fuzzy entropy, that tells us the indistinguishability of each study subject, patient or control, between baseline and outcome measurements. Two fuzzy entropy of similarity measures, one for patient and one for control, and two fuzzy entropy of symmetry of conditions measures, one for patient, one for control.

In our drug study example, each of $n$ patients receive drug ' $\mathrm{X}$ ' and each of $\mathrm{n}$ controls receive placebo ' $\mathrm{P}$ '. We have $\mathrm{n}$ patient control matches, each match providing 4 information granules. We have then $2 \mathrm{nX}$ and $2 \mathrm{nP}$ (where $\mathrm{X}$ stands for study drug patient and $\mathrm{P}$ for placebo patient) that must be compared.

For each patient who gets drug $\mathrm{X}$ we have $\mathrm{E} \operatorname{Sim}(\mathrm{A}, \mathrm{B})$ and $\mathrm{E} \operatorname{Symm}(\mathrm{A}, \mathrm{B})$, where $\mathrm{A}$ is the patient's state at baseline and $\mathrm{B}$ his/her state at outcome measurement.

For each control patient we have E Sim $(A, B)$ and $E$ Symm $(A, B)$ where $A$ is the control patient's state at the beginning of the trial and $\mathrm{B}$ is that state at the end of the trial.
This is non statistical information and each of the information granules is a value that falls in the unit interval. But our question is how did the patients receiving study drug change in elements and change in condition symmetry compared to those who got placebo? This information is again retrieved with our fuzzy entropy measures. How indistinguishable are the patient's who get the study drug from those who do not? Ultimately, we will then have information for two groups, and thus statistical information provided in the form of fuzzy entropy granules for similarity of elements at baseline and endpoint and symmetry of conditions at baseline and at endpoint.

So, for each matched pair of patient and control patients we can make a comparison:

E Sim $(A, B)$ patient and E Sim $(A, B)$ control and E Symm $(A, B)$ patient and E Symm $(A, B)$ control

We are now prepared to compare the change in clinical state elements and conditions taking place in the patient and in the control. Each of these entropy measures gives us a value in the unit interval. We now have a new pair of elements to make up another fuzzy set where each element is the entropy measure for the patient and that for the control. Table 1 gives an example.

We then want to know how dissimilar the patient's change was from the control's, first in terms of subsethood change E Sim A,B and for Symmetry change E Symm (A,B) and we calculate this for each pair of patients. These are the last two measure performed for each matched pair of patients and controls.

The formula is $\mathrm{E}(\operatorname{Sim} \mathrm{A}, \mathrm{B}$ pt, $\operatorname{Sim} \mathrm{A}, \mathrm{B}$ control $)=\mathrm{E}(\mathrm{E}$ $\operatorname{Sim}(\mathrm{A}, \mathrm{B}) \mathrm{pt}, \mathrm{E} \operatorname{Sim}(\mathrm{A}, \mathrm{B})$ control) for the subsethood comparison and $\mathrm{E}(\mathrm{Symm} A, \mathrm{~B}$ t, E Symm A,B control $)=\mathrm{E}(\mathrm{E}$ Symm A,B pt, E Symm A,B control).

We then have fuzzy entropy measures for all of the matched patient's and controls which tell us how indistinguishable the patient and matched control are in their response to drug versus placebo. We now can turn this into statistical information.

Finally, for $\mathrm{n}$ number of matched patients and controls, you can take the average of the entropy measures of similarity and symmetry to see how the population faired. This average entropy measure tells us how indistinguishable patient

Table 1. Experimental Comparison for Patients and Controls for Study drug X

\begin{tabular}{|c|c|c|c|}
\hline Elements BP and Blood Glucose as Fuzzy Set Elements & Patient & Control & Compare Patient Change to Control Change \\
\hline \hline A & $0.5,0.6$ & $0.7,0.8$ & \\
\hline B & $0.8,0.7$ & $0.3,0.4$ & \\
\hline F Sim (A,B) & 1.73 & 1.47 & \\
\hline K (A,B) & 1.60 & 2.14 & \\
\hline F Comp (A,B) & 1.13 & 0.33 & \\
\hline E Sim (A,B) & 0.67 & 0.22 & \\
\hline E Symm (A,B) & 0.39 & 0.22 & \\
\hline E Sim (pt,P) & & & \\
\hline Esym (pt, P) & & & 0.38 \\
\hline
\end{tabular}

Elements of each patient reflect blood pressure (BP) on the one hand and blood glucose on the other. A and B stand for fuzzy sets as points at baseline and at endpoint measurement of elements composing fuzzy sets as points and representing one patient pt receiving study drug or one control matched to that patient receiving placebo $\mathrm{P}$. 
and control groups are with regards to the change in the elements and the change in conditions between baseline and endpoint measures. The higher the entropy measures, the more alike the patient and control groups are in this regard and the less different the study drug group is from the placebo group.

For the two patients studied in our table above, we can say that when compared for change from baseline to outcome measurement our patient receiving drug and our patient receiving placebo were not at all very much alike in terms of how their elements changed and how their symmetry of conditions changed. This might be interpreted as a big difference made by the study drug.

More measures can be taken. We can ask, how much of the entropy measure for element change between patient on drug and patient on placebo is due to the indistinguishability between change in conditions. This measure is expressed as F Comp (pt, P) and is equal to E Sim (pt,P) -(E symm (pt,P)1 ) and is equal to $0.38-0.44+1=0.94$. So there is alot of indistinguishability between patient on drug and patient on placebo due to symmetry of conditions. This information can be turned into statistical information by taking the average values for both groups and performing the comparison. Taking our example, because our entropy measures are low as shown in Table 1, this means that whatever difference between study patients on drug and those on placebo there is, it is mainly attributable to study drug received.

The information presented by our fuzzy measures is not founded on probabilities and so is not based on chance. The significance of any of our fuzzy measures, such as those defining the difference between patients and controls in an experiment is therefore of a different nature than the $\mathrm{P}$ value of probability based statistics which tells us the probability that the results are based on chance. Through the measurement and accounting for different conditions, we hypothesize that the errors of evaluation in successive experiments or comparison of different patients that are due to different conditions will be limited by the use of fuzzy measurements of similarity and symmetry breaking.

\section{DISCUSSION}

In previous papers we defined a fuzzy measure of breaking symmetry of conditions and named it ' $\mathrm{K}$ ' [1]. We conceived of it as a measure of the role of unknown, unspecified and unmeasured factors, in changing the patient's state from A to B, each state represented by a fuzzy set as point in the unit hypercube where specified variables or set elements are valued in the unit interval $[1,7,8]$. Each state of one fuzzy set as point in the unit hypercube compared to another is a state of fuzzy subsethood between those states and which is distributed amongst the elements. The fuzzy entropy measure we develop called E Sim (A,B) tells us how indistinguishable the subsethood measures are for fuzzy sets as points $\mathrm{A}$ in $\mathrm{B}$ and $\mathrm{B}$ in $\mathrm{A}$.

As Riemann demonstrated, visualization in higher dimensions is key to understanding new concepts [13]. We employed the unit hypercube as a means of visualizing an indefinite number of dimensions [7]. The measure $\mathrm{K}$ was defined by us using the fuzzy Subsethood theorem [8]. This measure was initially designated an efficiency measure, and then a measure of extracubal causation where its measure is the size of outside dimensional force intruding into the fuzzy unit hypercube to cause the transition of fuzzy set A to fuzzy set $\mathrm{B}$. We have demonstrated using continuous cellular automata that $\mathrm{K}$ predicts symmetry breaking [9]. As supporting evidence that $\mathrm{K}$ is a measure of symmetry breaking, we demonstrated using continuous cellular automata, that the value of $\mathrm{K}$ is directly related to and can predict symmetry breaking. Our symmetry proof used a multiplicative transformation rule where $1 / \mathrm{K}$ is the initial condition and $\mathrm{K}$ the multiplier effect [9].

Symmetry in the unit hypercube is due to the unit interval. $\mathrm{K}$ breaks the unit interval and $1 / \mathrm{K}$ restores it. Thus we call $\mathrm{K}$ a measure of breaking of symmetry of conditions. When $\mathrm{K}$ acts it deforms, through breaking of symmetry of conditions and injection of unknown dimensions, the unit hypercube such that fuzzy set A becomes fuzzy set B. This can be demonstrated if each fuzzy set as point is considered a ball of the size of its cardinality. With the exchange of two balls of different size, all other fuzzy sets as ball points change their position to accommodate this change. Common to all the definitions of $\mathrm{K}$ is a potential dynamic between fuzzy sets for what is otherwise their static representation. The fuzzy entropy measure of symmetry $\mathrm{E}(\mathrm{K}, 1 / \mathrm{K})$ for transformation of fuzzy set as point A to B tells us the indistinguishability in extent of the break and restoration of symmetry of conditions

Symmetry of conditions in the probability space in the unit hypercube has unique properties regarding the dynamic of K. The fuzzy cardinality of each fuzzy set in the probability space is always $1[2,3]$. Symmetry of conditions, fuzzy cardinality of all sets as points in that space, are uniform. In the instance of the probability, the value of $\mathrm{K}$, the measure of breaking of symmetry of conditions, is also unchanged and always equal to 1 when any two fuzzy sets as points are interchanged. This is equaled in measure by $1 / \mathrm{K}$, the restoration of symmetry of conditions in the probability space. The measure of entropy of symmetry equals 1 in the probability space. This means that in the probability space symmetry of conditions remains unchanged reflecting the fact that in the move from fuzzy set A to B there is no net change in fuzzy cardinality even though for each unique transformation of fuzzy sets as points in the probability space there is a unique subsethood relation between A and B. This fuzzy subsethood relation is due alone then to change in element values of the fuzzy sets. In this circumstance, the change in value of $\mathrm{K}$ when multiple different fuzzy sets as points are considered is 0 . Otherwise the symmetry of conditions is broken.

When the symmetry of conditions is maintained, precise prediction is possible based on measures of interaction between the specified and measured fuzzy set elements. Our measure of fuzzy prediction demonstrates this fact for the probability space and is reflected by the fact that our fuzzy measure of entropy of similarity is maximum in probability. The degree to which the symmetry of conditions is broken corresponds to the degree of "fuzziness" of prediction possible and based on the elements alone given the transformation from A to B. In this case, causation is to a degree due to elements outside of those known and specified in the fuzzy set. For an unchanging value of $K$, say $K=1$, the role of the unknown in determining the change of the fuzzy set element 
values is the same and considered to be in practice non- existent. This is perfect fuzzy symmetry of conditions, but designated herein fuzzy because $\mathrm{K}$ is defined from the fuzzy Subsethood theorem [7]. It is this characteristic of probability that lends itself to scientific experimentation where repeated experiments can be performed under the same symmetry of conditions. Therefore, the type of certainty possessed by probability is the certainty that unknown, unmeasured conditions remain the same from trial to trial thus having no causal effect on trial outcome. Hence, prediction can be possible when rules governing the interaction of specified measured elements are known and other factors, conditions, do not have to be taken into account. These rules are those of probability theory and Aristotelian logic.

But, we continue to argue that there cannot be precise prediction based on probabilities alone in the case of the individual patient using the results of large double blind controlled randomized trials. Thus, we have sought another way to approach this problem with numerical precision. We chose to use fuzzy theory for this purpose because it was not bound by the rules and conditions of probability theory and because it is capable of precisiating ambiguities of patient uniqueness and expert decision, elements of the patient's unparalled conditions and physician perception [6]. Thus, in the clinical situation, it is possible to represent a patient by a fuzzy set where each element can be represented to its own extent and not constrained by the value of other elements [6]. In this instance, variables, each of which is present to a degree in the patient, are measured and through normalization or assignment by expert opinion given a value in the unit interval $[1,5,7]$. By considering patients as fuzzy "sets as points" in the fuzzy unit hypercube, where the sum of the elements of each fuzzy set are not required to add up to 1 , patients need not be confined to uniform conditions [9]. But, at present medical science assumes that it is impossible to compare the response of any two patients, for example, say to a given specified therapy, when the conditions of the patients are always different, unlike simple coin tosses. For this reason, scientific medical experimentation has confined itself to the use of probability theory and group statistics to determine medical efficacy. This state of affairs creates a paradox for the physician who must treat a patient with unique characteristics yet base his behavior on science.

It is exactly the comparison between individual patients or different states of the same patient over time which is the cognitive demand made of the physician when he or she builds up his or her clinical experience over time to develop expertise. It is this characteristic of medical decision making which has been called ambiguous because of the previous inability to precisiate it with numbers and "unscientific" because of the widely held belief that only comparisons can be made if conditions are exactly the same. These assumptions have in turn led to the mandate that clinical decisions are based on the results of large double blind controlled randomized trials performed within the rules of probability based statistics. But, the physician still must make his clinical decision for a single patient taking into account that patient's changing conditions. The application of the results of group studies to that patient is dependent on the physician's perception of how that patient is similar in known specified variables of the trial [16].
This paradox is solved by the fact that the construct of the fuzzy unit hypercube and consideration of patients as fuzzy sets as points allows for the visualization and measurement of changing patient variables and conditions [2, 5, 7]. In the real world, there is a dynamic non linear relation between changing variables and changing conditions. When these changes are measured, expert decision can be precisiated. Expert decision assumes that symmetry of conditions will be broken. Until the definition of $\mathrm{K}$, this dynamic could not be measured [1].

By using the measure of $\mathrm{K}$, it is possible to compare the amount of difference in change in conditions between any two fuzzy sets as points. Thus, serial changes of variable specified values within the same patient as when A transforms to B or comparison of two different patients designated by $\mathrm{A}$ and $\mathrm{B}$ can be directly compared taking into account difference in conditions. This can be done by calculating $\mathrm{K}$ for the given difference in fuzzy sets as points. Thus, single events in highly unknown and changing situations can be compared. The fuzzy measure of comparison can be used to do this. The fuzzy measure of similarity alone cannot do this because different symmetries of conditions are not accounted for, but using fuzzy similarity and comparison measures, a fuzzy entropy measure of similarity can be defined.

Dangson Tran has independently derived a discrete measure of symmetry of conditions for distributions [17]. His $\mathrm{K}=0$ for maintenance of symmetry of conditions in distributions and $\mathrm{K}>0$ up to infinity for progressive symmetry breaking up to complete randomness for the distributions when $\mathrm{K}=\infty$. Our fuzzy measure $\mathrm{K}$ is derived from the fuzzy Subsethood theorem for transition between single events in which symmetry of conditions is broken with $\mathrm{K}>1$ up to complete randomness when $\mathrm{K}=\infty$. Dangson argues convincingly that even in the case of probability distributions a $\mathrm{K}=\infty$ is never realized even using supercomputers and random number generation algorithms, i.e., perfect randomness is unattainable in the real world.

Randomness is related to causality and refers to the situations where unknown causes (elements outside of the measured elements of the fuzzy set and designated by K) have enough effect that symmetry of conditions decreases by degrees as randomness increases. In this situation, the causality attributable to the known measured variables decreases as the causality attributable to unknown unmeasured variables increases. So, causation in this situation is intimately related to breaking of symmetry of conditions. This state corresponds to low measures of fuzzy entropy of similarity and fuzzy entropy of symmetry as defined in this paper.

Fuzzy measures of similarity have been defined by others who use similar comparisons between fuzzy sets. However, these definitions do not take into account the difference in symmetry of conditions between fuzzy sets as points nor the dynamic of the degrees of breaking and restoration of that symmetry $[18,19]$. The fuzzy measures of comparison, predictability, and entropy of similarity and symmetry defined in this study do so. The argument of this paper derives from the geometric interpretation of fuzzy subsethood and fuzzy sets as 'sets as points' developed by Bart Kosko. The tools of the argument belong to those of the Generalized Theory of Uncertainty developed by Lotfi Zadeh. 


\section{CONCLUSION}

\section{Information and Symmetry}

The fuzzy measures of similarity and comparison as well as predictability defined in this paper come from the fuzzy Subsethood theorem which is the underlying conceptual structure of fuzzy theory. Subsethood is measured in cardinality, the measure space of fuzzy theory. We have shown here that fuzzy subsethood and cardinality provide the basic building blocks for the precise representation of causality to a degree [20].

The fuzzy measure of breaking of symmetry of conditions $\mathrm{K}$ is not dependent on the direction of change that takes place between fuzzy sets as points. Thus the path of change which fuzzy set elements take as they transform to new values is characterized by a "symmetrical" change in symmetry of conditions, where similarity of fuzzy sets as points and the measure of comparison between those points is unchanged. The fuzzy measure of prediction is also bidirectional and this is also true for the fuzzy entropy of this change. Thus, the path between two fuzzy sets is like a channel that carries information regarding the matching of patterns of known and unknown variables of two fuzzy sets. This channel is part of a dynamic structure of the unit hypercube that embodies the characteristics of change. We conclude that the fuzzy measures of similarity, comparison, prediction and entropies of similarity and symmetry provide a scientific representation and structure for experimentation when individual subjects or different states of the same subject are the object of study. Unlike probability, fuzzy theory, within the Generalized Theory of Uncertainty, breaking of symmetry of conditions, and a geometry of curvature such as that described by Riemann may be the new experimental tools for this science $[6$, $13,15]$.

\section{REFERENCES}

C. M. Helgason, and T. H. Jobe, " Perception -Based reasoning and fuzzy cardinality provide direct measures of causality sensitive to initial conditions in the individual patient", Int. J. Computat. Cogn., vol.1, no.2, pp. 99-104, June 2003.

[2] B. Kosko, Neural Networks and Fuzzy Systems. A Dynamical Approach to Machine Intelligence. Upper Saddle, NJ: Prentice Hall, 1992, pp. 274-5.
[3] B. Kosko, "Fuzziness vs probability", Int. J. Gen. Syst., vol 17, no.2, pp.211-240, March 1990.

[4] B. Kosko, Neural Networks and Fuzzy Systems. A Dynamical Approach to Machine Intelligence. Upper Saddle, NJ: Prentice Hall, 1992, pp. 274-5, 278-289.

[5] K. Burdzy, " Probability is symmetry. On the foundation of the science of probability”, Seattle, pp.1-98, 2003.

[6] L. A. Zadeh, " Toward a generalized theory of uncertainty (GTU)Principal concepts and ideas", Coomputational Statistics \& Data Analysis, vol. 51, issue 1, pp.1-40, November 2005.

[7] L. A. Zadeh, " Toward a theory of fuzzy systems", in Aspects of Network and System Theory, R.E. Kalman, N. DeClaris, Ed. Holt, Rinehardt and Winston, Inc., 1971, pp. 486.

[8] L. A. Zadeh, “Fuzzy sets”, Inform Cont., vol. 8, no.3, pp.338-353, June1965.

[9] C. M. Helgason, and T. H. Jobe, " The fuzzy cube and causal efficacy: representation of concomitant mechanisms in stroke", Neural Netwo., vol. 11, no.3, pp. 549-555, April 1998.

[10] B. Kosko, Neural Networks and Fuzzy Systems. A Dynamical Approach to Machine Intelligence. Upper Saddle, NJ: Prentice Hall, 1992, pp. 269-273.

[11] B. Kosko, Neural Networks and Fuzzy Systems. A Dynamical Approach to Machine Intelligence. Upper Saddle, NJ: Prentice Hall, 1992, pp. 278.

[12] C. M. Helgason, and T. H. Jobe, " Fuzzy logic and continuous cellular automata in warfarin dosing of stroke patients", Curr. Treat. Options Cardiovasc. Med., vol. 7, no.3, pp. 211-218, March 2005.

[13] G. B. Riemann, " On the Hypotheses which lie at the foundation of Geometry, 1854. Translation by Henry H White, in A Source Book for Mathematics, David Eugene Smith, Ed. New York: McGrawHill, 1929, pp. 411-425.

[14] B. Kosko: Neural Networks and Fuzzy Systems. A Dynamical Approach to Machine Intelligence. Upper Saddle, NJ: Prentice Hall, 1992, pp. 275-278.

15] J. Rosen, Symmetry in Science: An Introduction to the General Theory, New York: Springer Verlag, 1995.

[16] D. P. Foster, and H. P. Young, "On the impossibility of predicting the behavior of rational agents", Proc. Natl. Acad. Sci. USA, vol. 98, no.22, pp. 12848-12853, October 2001.

[17] D. Tran, Symmetry and The End of Probability. Second edition. San Jose: Last Science Publishing, 2003.

[18] J. T. Rickard, J. Aisbett J, and G. Gibbon, "Knowledge representation and reasoning in conceptual spaces", in Proceedings of the 2007 IEEE Symposium on foundations of Computational Intelligence (FOCI 2007), pp. 583-590, April 2007.

[19] W. L. Hung, and M. S. Yang, "Similarity measures of intuitionistic fuzzy sets based on Lp metric", Int. J. Approx. Reason., vol. 46, no.1, pp. 109-119, September 2007.

[20] J. Cat, " Fuzzy empiricism and fuzzy set causality: what is all the fuzzy about?”, Philos. Sci., vol. 73, pp 26-41, January2006. 